\title{
SYSTEMIC SCLEROSTIN ANTIBODY TREATMENT INCREASES OSSEOINTEGRATION AND BIOMECHANICAL COMPETENCE OF ZOLEDRONIC-ACID-COATED DENTAL IMPLANTS IN A RAT OSTEOPOROSIS MODEL
}

\author{
P. Korn ${ }^{1, *}$, I. Kramer' ${ }^{2}$, F. Schlottig ${ }^{3}$, N. Tödtmann ${ }^{4}$, U. Eckelt ${ }^{10}$, A. Bürki ${ }^{5}$, S.J. Ferguson ${ }^{6}$, A. Kautz ${ }^{7}$ \\ M. Schnabelrauch ${ }^{7}$, U. Range ${ }^{8}$, M. Kneissel ${ }^{2}$ and B. Stadlinger ${ }^{9}$
}

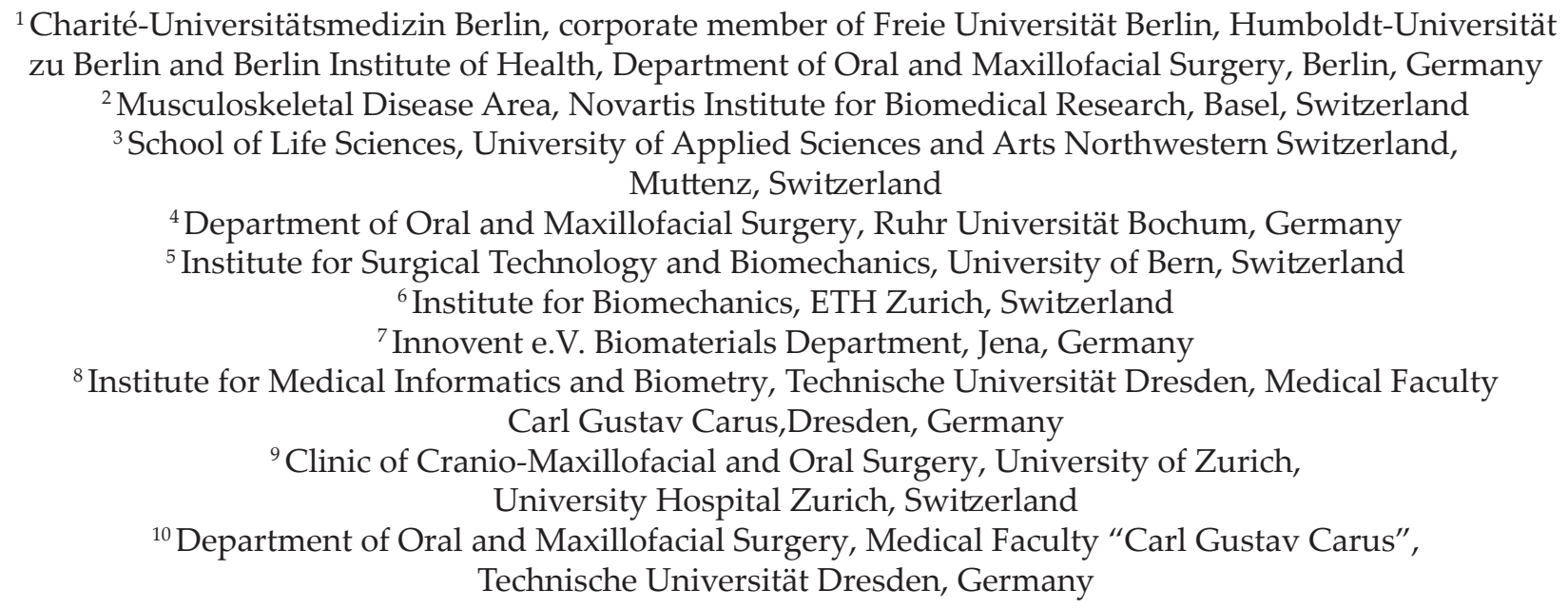

\begin{abstract}
Osseointegration of dental implants can be promoted by implant-surface modifications using bisphosphonate coatings. In addition, it is of clinical interest to promote peri-implant bone formation and to restore bony structure in low bone-mass patients. The present study evaluated a combination of an anti-resorptive zoledronic acid (ZOL) implant-coating and a systemically applied sclerostin antibody, a known bone anabolic treatment principle, versus sole sclerostin antibody treatment or ZOL implant-coating in a rat osteoporosis model.

Uncoated reference surface implants or ZOL-coated implants ( $n=64 /$ group) were inserted into the proximal tibia of aged osteoporotic rats three months following ovariectomy. 32 animals of each group received once weekly sclerostin antibody therapy. Osseointegration was assessed 2 or 4 weeks post-implantation by ex vivo $\mu \mathrm{CT}$, histology and biomechanical testing.

Overall implant survival rate was $97 \%$. Histomorphology revealed pronounced bone formation along the entire implant length of ZOL-coated implants. At 4 weeks following implant insertion, bone-implant contact, cancellous bone mineral density and bone volume/tissue volume were significantly increased for the combination of ZOL and sclerostin antibody as compared to sclerostin antibody or ZOL implant-coating alone. Removal torque was also significantly increased in the combination therapy group relative to animals receiving only sclerostin antibody therapy or ZOL-coated implants.

In an osteoporotic rat model, the combination of anti-resorptive ZOL implant-coating and systemically applied sclerostin antibody led to significantly increased peri-implant bone formation. Therefore, the combination of ZOL and the osteoanabolic sclerostin antibody was more effective than either agent alone.
\end{abstract}

Keywords: Dental implant, osseointegration, osteoporosis animal model, sclerostin antibody, zoledronic acid.

*Address for correspondence: Paula Korn, Department of Oral and Maxillofacial Surgery, CharitéUniversitätsmedizin Berlin, corporate member of Freie Universität Berlin, Humboldt-Universität zu Berlin and Berlin Institute of Health, Augustenburger Platz 1, 13353 Berlin, Germany.

Telephone number: +49 30450555022 Email: paula.korn@charite.de

Copyright policy: This article is distributed in accordance with Creative Commons Attribution Licence (http://creativecommons.org/licenses/by-sa/4.0/). 


\section{Introduction}

Long-term survival of dental implants depends on the mechanically stable fixation of an implant in the host bone (Branemark et al., 1977). Conditions affecting bone quality and quantity, such as osteoporosis, may limit an implant's clinical success (Ihde et al., 2009; Scully et al., 2007). Postmenopausal osteoporosis is the most common type of osteoporosis. In Germany, it has a prevalence of about $59 \%$ in women over 75 years and leads to reduced bone mass and increased bone fragility (Haussler et al., 2007). In the USA, osteoporosis prevalence is $52 \%$ in women over 80 years (Looker, 1995). Therefore, it could be hypothesised that dental implant loss would occur frequently in such patients with reduced bony template. Yet, apart from a positive correlation between systemic and alveolar bone loss (Jeffcoat, 2006), no increased malfunction of dental implants has been reported to date (Holahan et al., 2008; Marco et al., 2005).

Drugs for the treatment of osteoporosis are aimed at inhibiting ongoing catabolic bone resorption and/or promoting anabolic bone formation. It is currently unclear whether such drugs would also positively influence implant osseointegration and biomechanical competence. Clinically, bisphosphonates are commonly applied for the inhibition of osteoclastic bone resorption and bone loss in osteoporosis (Crandall et al., 2014). Zoledronic acid (ZOL) is an intravenously applied potent bisphosphonate commonly prescribed to patients suffering from postmenopausal osteoporosis. ZOL has a high affinity to mineralised bone tissue, where it inhibits the activity of bone-resorbing osteoclasts through suppression of farnesyl diphosphate synthase enzymatic activity (Lin, 1996). This leads to irreversible inhibition of osteoclast function and reduced osteoclast number.

Both types of bone cells, osteoblasts and osteoclasts, also play a major role in peri-implant bone healing. Osseointegration of dental implants can be altered by several surface modifications, such as roughening or application of implant coatings with components of the extracellular matrix (Stadlinger et al., 2008; 2009), or with pharmaceutical agents. Bisphosphonates have been used as implant coatings in preclinical (Andersson et al., 2010; Gao et al., 2009; Stadlinger et al., 2013; Wermelin et al., 2007; Wermelin and Aspenberg et al., 2008) as well as clinical studies (Abtahi et al., 2010). Ovariectomised (OVX) rats are oestrogen-deprived and serve as an established model of postmenopausal osteoporosis. In this animal model, bisphosphonate-coated implants show a significantly higher bone-implant contact (BIC) and bone-volume density (BVD) as compared to reference surface implants (Stadlinger et al., 2013). In addition, other osteoporosis drugs, such as oestrogen (Duarte et al., 2005; Qi et al., 2004), strontium ranelate (Maimoun et al., 2010) or parathyroid hormone (Shirota et al.,
2003), are under preclinical investigation regarding their potential to promote osseointegration. Another promising emerging approach to osteoporosis therapy is the application of monoclonal sclerostinblocking antibodies. Sclerostin is a small secreted glycoprotein and a potent inhibitor of the bone anabolic canonical WNT/ $\beta$-catenin signalling pathway (Baron and Kneissel, 2013; Delgado-Calle et al., 2017). It is predominantly expressed by osteocytes, terminally differentiated cells of the osteoblastic lineage. It binds to the canonical WNT co-receptors lipoprotein receptor-related protein-4 (LRP4), LRP5 and LRP6, thereby, inhibiting bone formation of osteoblasts (Leupin et al., 2011; Poole et al., 2005). Additionally, osteoclast activity is increased as a result of sclerostin-mediated inhibition of WNT signalling (Delgado-Calle et al., 2017). Sclerostin loss-of-function in humans and animals leads to a large bone mass phenotype associated with normal bone quality. Sclerostin antibodies block the binding of sclerostin to LRP5/6 WNT signalling co-receptors, thereby resulting in elevated osteoblastic WNT signalling activity, which leads to increased bone formation and reduced bone resorption. Numerous animal studies have demonstrated the osteoanabolic effect of sclerostin antibodies. For example, in OVX rats, weekly application of sclerostin antibody leads to a significant improvement in bone mass and architecture after 12 months of observation (Ominsky et al., 2017). Similarly, it is of clinical interest whether sclerostin antibodies also promote osseointegration of dental implants, enabling a functional and aesthetic rehabilitation after tooth loss. Tooth extractions, e.g. due to caries or periodontitis, increase with age. Furthermore, many patients suffer from systemic diseases such as e.g. osteoporosis. Thus, the combination of systemic bone anabolic sclerostin antibody treatment for bone restoration with an implant surface coating for the improvement of peri-implant bone healing under compromised bone conditions may hold the potential to increase the number of patients that can benefit from dental implants.

The present study evaluated the effect of systemic sclerostin antibody application and ZOL implant coating on peri-implant bone formation in a rat osteoporosis model. The hypothesis was that the combination of sclerostin antibody application and ZOL implant-coating increased the peri-implant bone formation to a greater extent than either therapeutic principle alone.

\section{Materials and Methods}

\section{Animals}

The study experimental protocol and the animal care conformed to the Swiss federal law for animal protection under the control of the Basel-Stadt Cantonal Veterinary Office, Switzerland. 128 
6-month-old female virgin Wistar rats underwent bilateral ovariectomy under general anaesthesia. 14 weeks after surgery, the animals were randomised into two groups (64 rats/group) based on an even distribution of proximal tibial bone mineral density (BMD), as assessed by micro-computed tomography $(\mu \mathrm{CT})$, and body weight as first and second rank parameters, respectively. Then, each animal received one implant in the left proximal tibia metaphysis. After implant insertion, 32 rats of each group received $100 \mathrm{mg} / \mathrm{kg}$ sclerostin antibody applied intravenously once weekly until sacrifice. Animals were further randomised into two sub-groups (16 rats/sub-group) and sacrificed either 2 or 4 weeks following implant insertion, respectively (Fig. 1). Tibial bone samples from 8 animals per sub-group were assessed by $\mu C T$ and histomorphometry. The additional 8 samples were utilised for biomechanical testing.

\section{Implants}

Experimental-threaded titanium implants (grade 4), with major diameter $1.7 \mathrm{~mm}$, minor diameter $1.1 \mathrm{~mm}$ and length $3.0 \mathrm{~mm}$, were used (Thommen Medical AG, Waldenburg, Switzerland). The head of the screw was of cylindrical shape to allow for coaxial alignment and equipped with an inverse slot drive. The surface of all implants was sandblasted and thermally acid-etched. The $S_{a}$ value following this procedure is approximately $2.0 \mu \mathrm{m}$ (Stadlinger et al., 2012a). These implants with reference surface $(n=64)$ were inserted in one experimental group.
An additional 64 implants were coated with a ZOLstearate complex using $8.5 \mu \mathrm{g}$ of ZOL acid per implant in a spray-coating process, as previously described (Stadlinger et al., 2013). All implants were sterilised by gamma-radiation before insertion.

\section{Implant surgery}

Animals were anaesthetised with $60 \mathrm{mg} / \mathrm{kg}$ ketamine and $8 \mathrm{mg} / \mathrm{kg}$ xylazine by intraperitoneal injection and received $0.05 \mathrm{mg} / \mathrm{kg}$ buprenorphine subcutaneously prior to implantation and $0.03 \mathrm{mg} / \mathrm{kg}$ twice daily for $2 \mathrm{~d}$ post-implantation for analgesic purposes. All operative procedures were performed under sterile conditions. For implant placement, a $15 \mathrm{~mm}$ longitudinal incision was made along the medial side of the left tibia and the musculo-periosteal flap was lifted, as described previously (Stadlinger et al., 2013). A conical drill with a diameter of 1.1-1.5 mm was used under water-cooling for osteotomy $2 \mathrm{~mm}$ distal to the growth plate of the proximal tibia metaphysis. Implants were placed using an implant screwdriver. After placement, a rounded polyetherether-ketone healing cap was placed on the cuboid implant top. The soft tissue was repositioned and sutured in two layers, using resorbable sutures (Safil $^{\circledR} 6 \times 0$, Braun, Melsungen, Germany). An aerosol bandage was applied (Flint MED, TogalWerk, München, Germany) and wound healing was controlled daily for the first week post-implantation and twice per week during the following healing period. All animals received fluorochrome markers

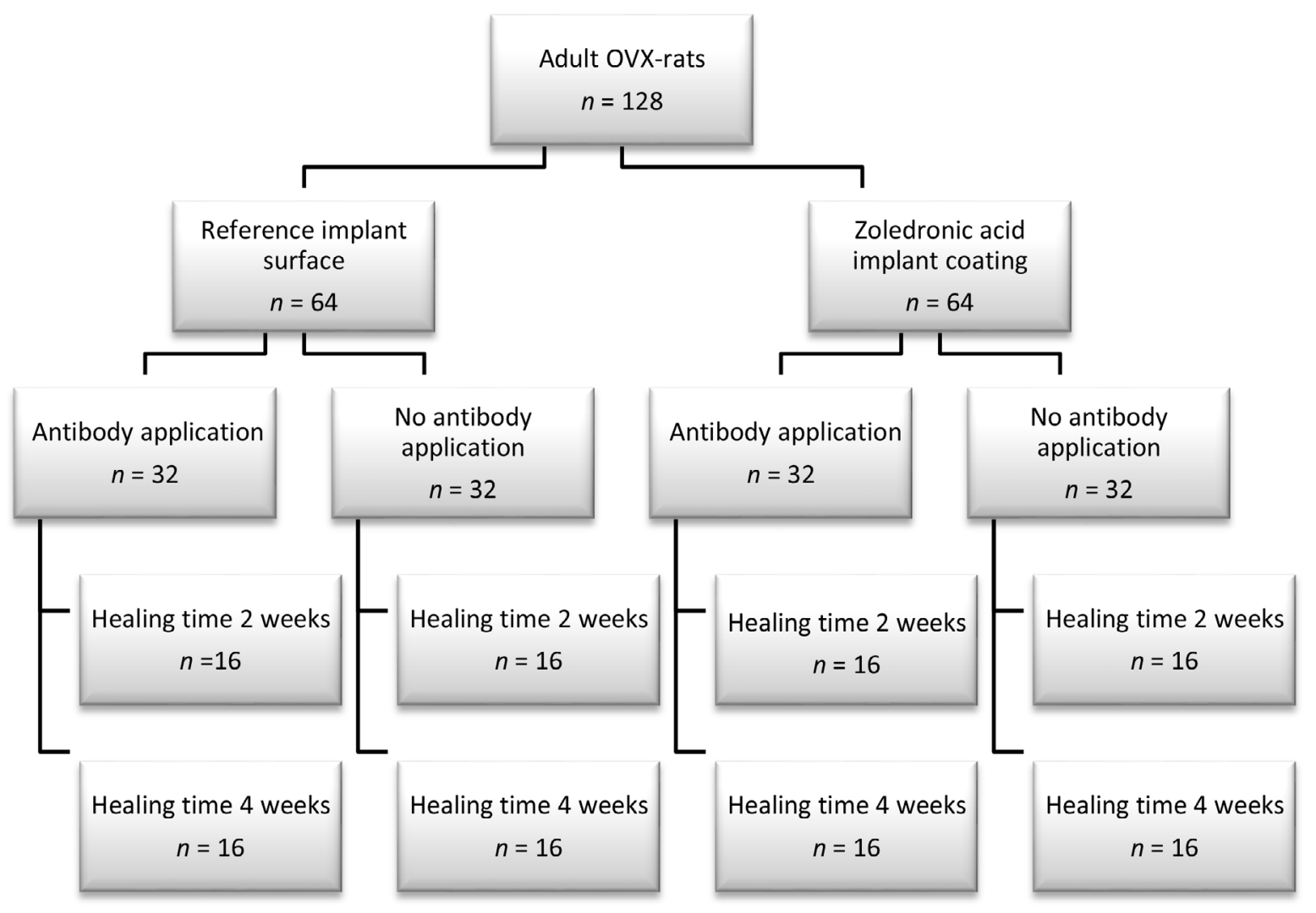

Fig. 1. Study design. After sacrifice, 8 samples per group were analysed by $\mu \mathrm{CT}$ and histology and the other 8 samples were used for biomechanical testing. 
by subcutaneous injection $10 \mathrm{~d}$ (alizarin complexone, $20 \mathrm{mg} / \mathrm{kg}$, Merck) and $3 \mathrm{~d}$ (calcein, $30 \mathrm{mg} / \mathrm{kg}$, SigmaAldrich) prior to sacrifice.

124 of 128 rats completed the study, which represented an overall implant survival rate of $97 \%$. 4 animals died during surgery or post-operative healing. Rats exhibited normal behaviour and no signs of abnormal wound healing or other overt phenotypes were observed.

\section{Tissue processing}

After sacrifice, the left tibiae were removed and the proximal halves were fixed in Schaffer's solution for $24 \mathrm{~h}$ at $4{ }^{\circ} \mathrm{C}$, followed by storage in $70 \%$ ethanol. Following ex vivo $\mu \mathrm{CT}$ analysis, the samples were dehydrated and embedded in methylmethacrylate to generate $50 \mu \mathrm{m}$-thick ground sections. These were cut in parallel to the implant length axis and perpendicular to the tibial axis, as described previously (Donath and Breuner, 1982). Fluorescent microscopy was performed prior to Masson-Goldner trichrome staining.

\section{Computed tomography}

For even distribution of the animals into groups, cross-sectional BMD was evaluated in the left proximal tibia metaphysis by peripheral quantitative computed tomography (pQCT, Norland XCT-2000; Stratec, Pforzheim, Germany) prior to implant surgery (fitted with an Oxford 50 AM X-ray tube and a $1 \mathrm{~mm}$ diameter collimator; voxel size: $0.2 \mathrm{~mm} \times 0.2 \mathrm{~mm} \times 1 \mathrm{~mm}$; scan speed: scout view $20 \mathrm{~mm} / \mathrm{s}$; final scan: $10 \mathrm{~mm} / \mathrm{s}, 1$ block, contour mode 1, peel mode 2; threshold: $610 \mathrm{mg} / \mathrm{cm}^{3}$ ).

Ex vivo, $\mu \mathrm{CT}$ measurements were performed with a $\mu \mathrm{CT} 40$ instrument [Scanco Medical; voxel size: $10 \mu \mathrm{m}$; 192 slices; energy: $70 \mathrm{kV}, 114 \mu \mathrm{A}$; high resolution; integration time: $300 \mathrm{~ms}$; cone beam continuous rotation] as previously described (Rebaudi et al., 2004). Bone and titanium were distinguished using the appropriate Gaussian filters (sigma 1.2, bone; 2.0, titanium; support 2, bone 3, titanium) and threshold procedures (bone: 370-700; titanium implant: 701-1000). BMD and bone volume/ tissue volume $(\mathrm{BV} / \mathrm{TV})$ were determined in the cancellous bone region surrounding the implant ( 3 to 10 voxels distance from the implant) and in the region adjacent to it (10 to 23 voxels distance from the implant). Since results did not differ between the two regions, data were merged for the results presented in Fig. 5 and reflected a total width of $200 \mu \mathrm{m}$. In addition, BIC was determined.

\section{Histomorphometry}

Fluorescence microscopy was performed at up to $40 \times$ magnification (Olympus BX 61). Polyfluorochrome labels were qualitatively analysed for bone growth dynamics, location and label sequences. Subsequently, Masson-Goldner-trichrome-stained sections were imaged following alignment using a motorised measuring stage (Märzhäuser, Wetzlar, Germany) for multiple alignment scanning connected to a computerised system for histomorphometry (Cell ${ }^{\wedge} \mathrm{F}$, Imaging Software for Life Science, Olympus). BIC was measured in the cancellous bone compartment along the implant surface. The apex of the implant was not included. Cancellous bone area per tissue area (BA/ TA) was measured in a region adjacent to the implant and in the surrounding area, as described previously (Stadlinger et al., 2013).

\section{Biomechanical evaluation}

64 specimens (8 implants per group and healing time) underwent biomechanical testing at the day of harvest. Healing caps were removed and the tibiae were embedded in dental plaster (Fujirock EP, Improved type 4 dental stone, GC, Leuven, Belgium) using a custom potting device, which provided coaxial alignment of implant and actor of the testing machine. Specimens were mounted on a servohydraulic testing machine (MTS 858 Mini Bionix, MTS Systems Corporation, Minneapolis, MN, USA) equipped with a $2 \mathrm{Nm}$ load cell and signal amplifier (accuracy 2 Nmm; D2209; Lorenz Messtechnik, Alfdorf, Germany). A bellow clutch was used to compensate axial motion and a claw coupling to ensure neutral initial fixation (no moment). Implants were rotated counter-clockwise at the constant rate of $0.5 \%$ s until peak moment was passed and the residual friction moment was clearly reached. Moment and angle data were recorded for subsequent data analysis at a sampling rate of $50 \mathrm{~Hz}$. Removal torque (RT: Nmm) was defined as the maximal torque and interfacial stiffness $\left(\mathrm{Nmm} /{ }^{\circ}\right)$ was calculated by linear interpolation of the moment-angle curve between $20 \%$ to $80 \%$ of RT (MATLAB R2008, The MathWorks, Natick, MA, USA).

\section{Statistical analysis}

Data are presented as mean values \pm standard deviation. Data distribution was tested by Kolmogorov-Smirnov test. Variance analysis of all groups by ANOVA with Bonferroni-adjusted multiple comparisons of mean values was performed. The level of significance was set to $p=0.05$ in all statistical tests. Statistical analyses were performed by SPSS for Windows ${ }^{\circledR} 25$ (SPSS, Chicago, IL, USA) or Prism 7 (GraphPad Inc., La Jolla, CA, USA).

\section{Results}

\section{Fluorochrome marker uptake}

Calcein labels were readily detectable. They were more pronounced in the 2 -week healing groups than in the 4-week healing groups independent of the implant surface (Fig. 2). In addition to the incorporation of fluorochrome labels into the unaffected secondary spongiosa and endosteum at sites of bone formation and mineralisation related to normal bone turnover, a large amount of intensive calcein labelling was detectable in the peri-implant 
Healing time 2 weeks

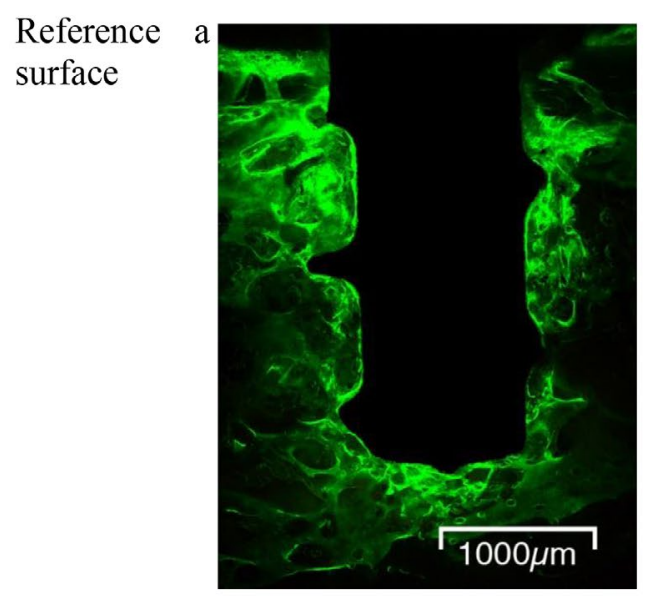

ZOL-
implant
coating
Healing time 4 weeks
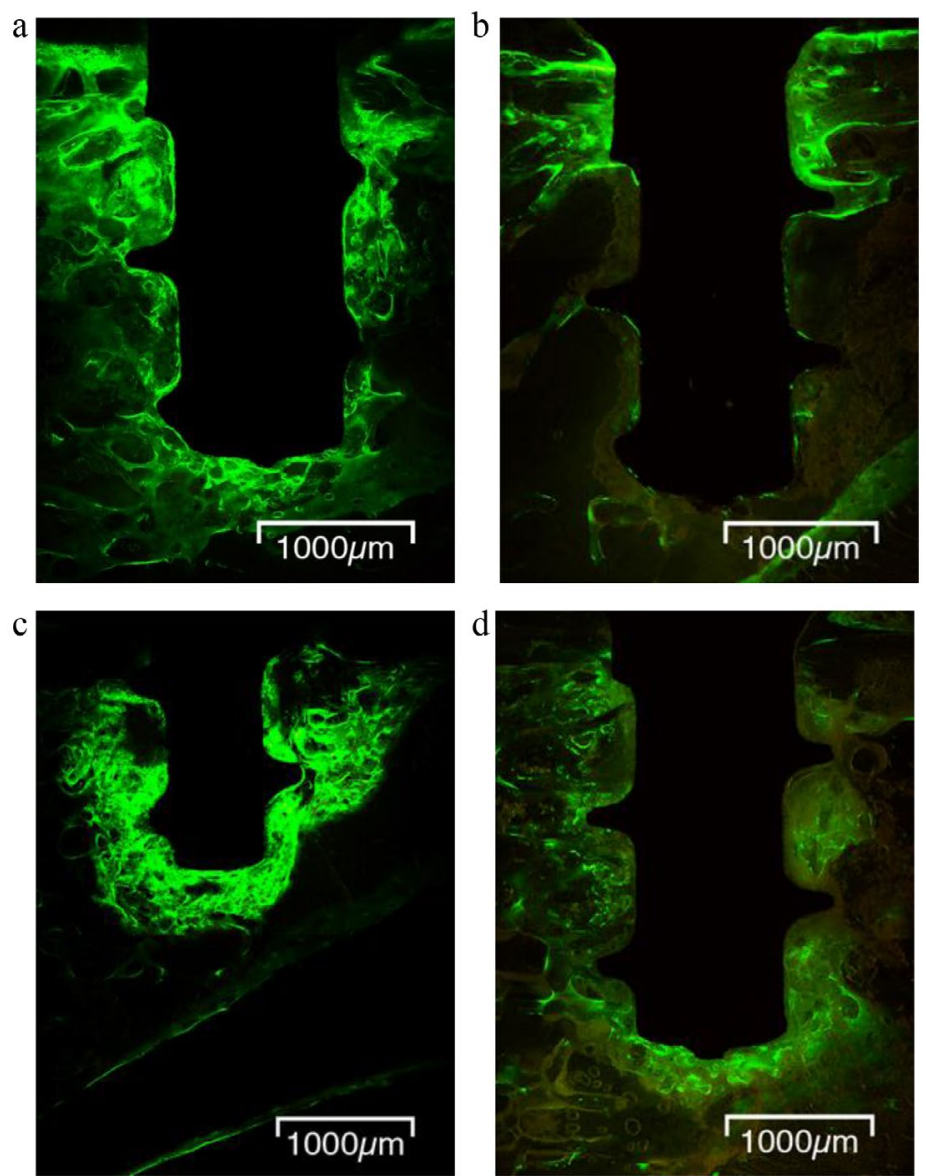

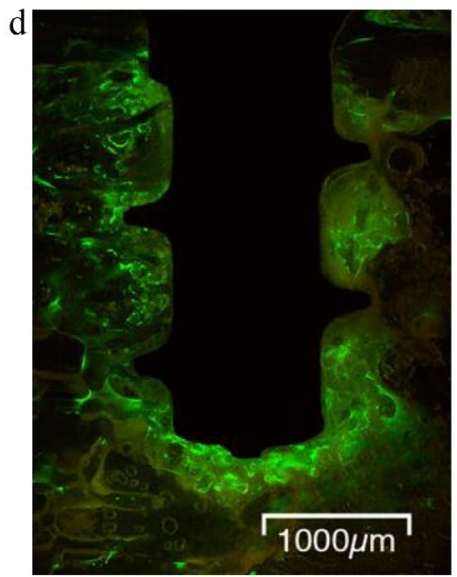

Fig. 2. Fluorescence microscopy. Multiple image alignment: representative images of $(\mathbf{a}, \mathbf{b})$ reference implants and (c,d) ZOL-coated implants, $(\mathbf{a}, \mathbf{c}) 2$ and $(\mathbf{b}, \mathbf{d})$ 4 weeks post-implantation. Sclerostin antibody was applied in both groups once weekly. tissue up to $1 \mathrm{~mm}$ distant from the implant surface in both groups. Alizarin red, which was applied before calcein, was generally rarely visible in the peri-implant area at either time point (Fig. 2).

After 2 weeks of implant insertion and weekly sclerostin antibody application, reference implants showed calcein labelling adjacent to the implant surface, whereas ZOL-coated implants were characterised by a more extensive dissemination in the peri-implant area (Fig. 2a,c). With increasing healing time, a decrease in fluorochrome marker uptake was detectable in all experimental groups.

After 4 weeks and ongoing sclerostin antibody application, ZOL-coated implants still revealed homogenous bone formation and mineralisation surrounding the whole implant. In contrast, in rats that received reference surface implants, calcein labels were mostly restricted to the cortical bone area, whereas there was little label detectable around the threads (Fig. 2b,d).

\section{Histomorphology}

2 weeks after implant insertion, little peri-implant bone was present in rats that received reference surface implants only and the implants were mainly fixed by cortical bone (Fig. 3a). With ongoing time, peri-implant bone loss was more pronounced (Fig. $3 b)$. In contrast, rats that received reference surface implants but combined with sclerostin antibody application showed much more cancellous bone in the peri-implant area, being mainly located on the proximal tibial site of the implants 2 weeks after implantation (Fig. 3c). Moreover, distant osteogenesis was detectable in this group in line with the systemic action of the applied sclerostin antibody. However, 4 weeks following implant insertion, cancellous bone was reduced and the lamellar bone-implant contact was predominantly restricted to the tip of the implant (Fig. 3d).

The combination of sclerostin antibody application and ZOL implant-coating led to pronounced bone formation along the entire implant length. Immature woven bone was located within the implant threads whereas trabeculae of mature lamellar bone could be observed more distantly (Fig. 3g). With increasing healing time, bone maturation was observed as expected, leading to a homogenous trabecular structure and trabeculae in contact with implant surfaces (Fig. 3h). When compared with the referencesurface-coated implants, ZOL-coated implants were associated with more woven bone in the peri-implant area and the conversion to mature cancellous lamellar bone was less pronounced after 4 weeks of healing (Fig. 3e-h).

\section{BIC analysed by histomorphometry}

Implants with reference surface and antibody treatment showed a BIC of $33.2 \pm 18.5 \%$ after 2 weeks 
of healing, which decreased non-significantly to $24.1 \pm 9.7 \%$ after 4 weeks (Fig. 4 a). 2 weeks postimplantation, the BIC around ZOL-coated implants with additional antibody application was comparable to that of reference surface implants with combined sclerostin antibody therapy (Fig. 4a). In contrast, after 4 weeks of healing, the BIC further increased significantly to $57.4 \pm 15.0 \%$ in rats with ZOL-coated implants and sclerostin antibody treatment. This was significantly different from the reference groups with antibody treatment only. Comparison among groups without antibody application revealed a significantly increased BIC for ZOL-coated implants after 4 weeks.

\section{BA/TA analysed by histomorphometry}

All experimental groups had a higher BA/TA adjacent to the implant surface as compared to the distant area (Fig. $4 \mathbf{b}, \mathbf{c})$. However, relative to reference groups and irrespective of antibody treatment, ZOL-coated implants showed increased BA/TA adjacent to the implant at all time points, reaching significance at 4 weeks of healing with $(32.3 \pm 11.5 \%$ for ZOL-coated implants versus $10.9 \pm 4.4 \%$ for reference-surfacecoated implants) and without combined sclerostin antibody treatment $(23.8 \pm 8.6 \%$ for ZOL-coated implants versus $4.5 \pm 4.2 \%$ for reference-surfacecoated implants), respectively (Fig. 4 b). In the

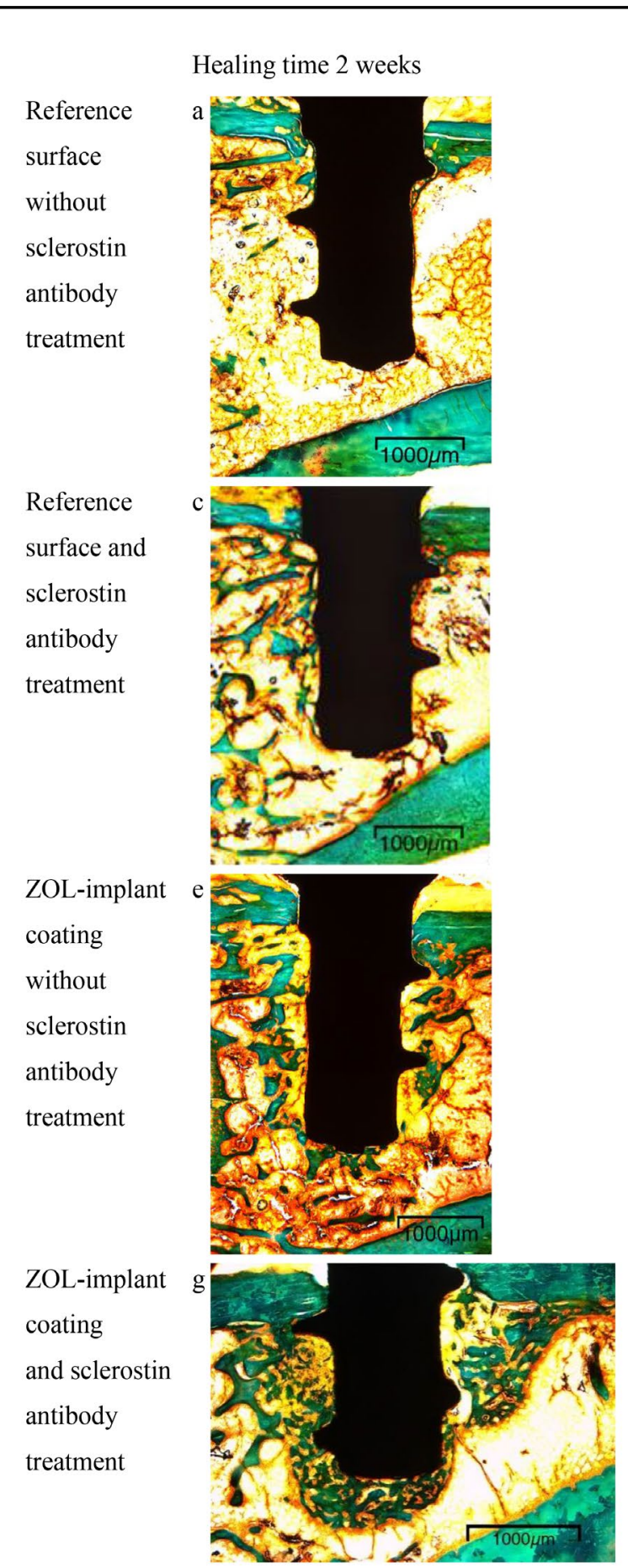

Healing time 4 weeks

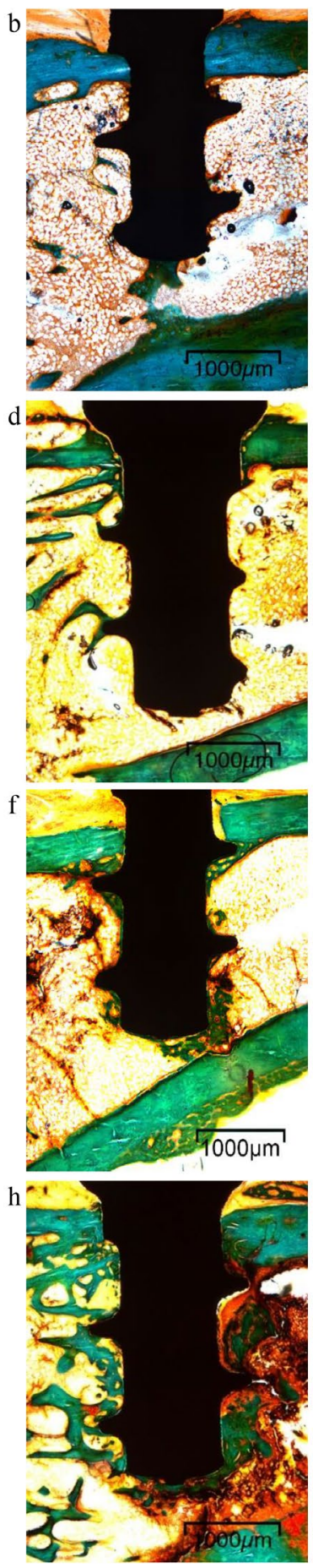

Fig. 3. Histology. MassonGoldner trichrome staining; multiple image alignment: representative images of $(\mathbf{a}, \mathbf{b}, \mathbf{c}, \mathbf{d})$ reference implants and $(\mathbf{e}, \mathbf{f}, \mathbf{g}, \mathbf{h})$ ZOL-coated implants, $(\mathbf{a}, \mathbf{c}, \mathbf{e}, \mathbf{g}) 2$ and $(\mathbf{b}, \mathbf{d}, \mathbf{f}, \mathbf{h}) 4$ weeks postimplantation. (c, d, g,h) Sclerostin antibody was applied in both groups once weekly. 
distant area, the combination of ZOL coating and antibody application led to a significantly increased BA/TA at 4 weeks post-implantation as compared to reference-coated implants without sclerostin antibody treatment (Fig. 4c).

\section{BIC analysed by $\mu \mathrm{CT}$}

Without antibody application or ZOL implantcoating, BIC decreased non-significantly during healing (Fig. 5a). After 4 weeks, the highest BIC was detected for ZOL-coated implants with antibody treatment $(60.0 \pm 2.5 \%)$, which was followed by ZOL-coated implants without antibody application $(47.8 \pm 10.4 \%)$, albeit the difference between these two groups did not reach statistical significance. However, the difference between the ZOL-coated implant groups and the reference implant groups was statistically significant at the 4-week time point (Fig. 5a). Overall, these findings were in line with the histomorphometry results.

\section{Cancellous bone mass analysed by $\mu \mathrm{CT}$}

The relative cancellous bone volume $(\mathrm{BV} / \mathrm{TV})$ as well as cancellous BMD were significantly increased for ZOL-coated implants at 4 weeks postimplantation (Fig. 5b,c). 4 weeks post-implantation, $\mathrm{BV} / \mathrm{TV}$ measured for the reference surface group was
$4.7 \pm 3.5 \%$, whereas it was 6 times higher $(31.0 \pm 7.6 \%)$ for the ZOL-coated surface group with sclerostin antibody treatment. Likewise, the combination of implant coating and antibody administration led to an almost 2-fold increase in BMD as compared to the reference surface group after 4 weeks of healing (Fig. 5c). The comparison of the two experimental groups with antibody treatment revealed obvious differences regarding the dynamic development of cancellous bone mass. Implants with reference surface displayed a decrease in BV/TV and BMD with proceeding healing time, whereas the coating with ZOL promoted bone mass and density increases (Fig. 5b,c).

The analysis of the trabecular thickness revealed comparable results for groups which had received the sclerostin antibody treatment. Both sclerostinantibody-treated groups displayed higher trabecular thickness as compared to groups without sclerostin antibody treatment (Fig. 5d).

\section{Biomechanical properties}

Removal torque as well as stiffness were comparable after 2 weeks of healing (Fig. 6). Irrespective of the implant surface coating, both parameters increased with healing time, but ZOL-coated implants displayed a higher removal torque at 4 weeks a

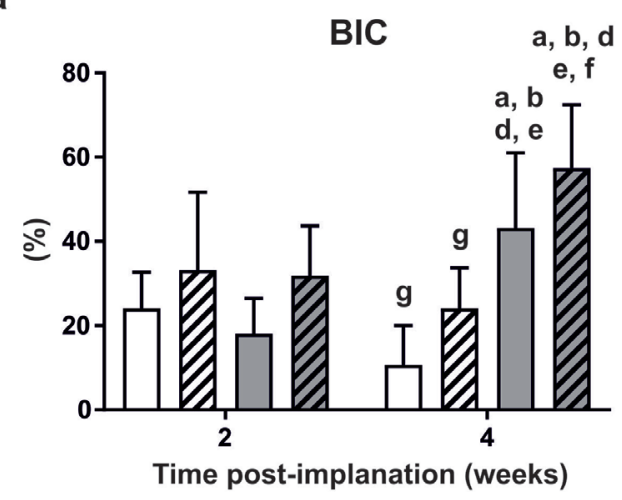

C

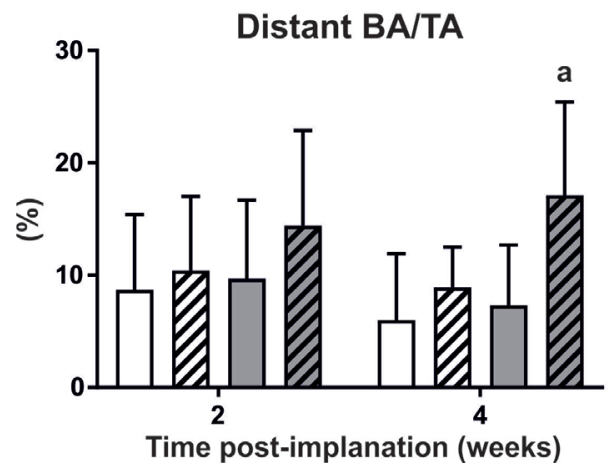

b

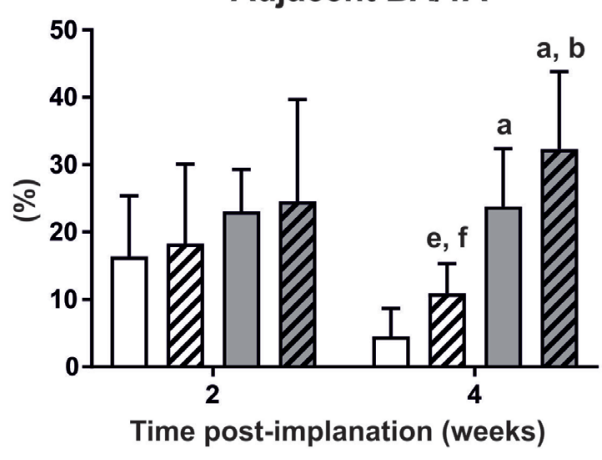

$\square$ Reference coating without sclerostin antibody
$\square$ Reference coating with sclerostin antibody
$\square$ zOL coating without sclerostin antibody
$\square$ ZOL coating with sclerostin antibody

Fig. 4. Histomorphometry parameters 2 and 4 weeks post-implantation. (a) BIC, (b) adjacent and (c) distant BA/TA were quantified. Statistical significance is designated as follows: ${ }^{a} p<0.05$ versus reference coating without sclerostin antibody at same time point; ${ }^{\mathrm{b}} p<0.05$ versus reference coating with sclerostin antibody at same time point; ${ }^{c} p<0.001$ versus reference coating without sclerostin antibody at 2 weeks post-implantation; ${ }^{\mathrm{d}} p<0.05$ versus reference coating with sclerostin antibody at 2 weeks post-implantation; e $p<0.05$ versus ZOL coating without sclerostin antibody at 2 weeks post-implantation; ${ }^{\mathrm{f}} p<0.05$ versus ZOL coating with sclerostin antibody at 2 weeks post-implantation. 
post-implantation when compared with reference implants. After 4 weeks, the combination of ZOLcoating and sclerostin antibody application led to a significantly increased removal torque as compared to antibody treatment only (Fig. 6a). With exception of the statistically increased stiffness of referencecoated implants at 4 as compared to 2 weeks postimplantation, no statistically significant differences were measurable for implant interface stiffness (Fig. $6 b)$.

\section{Discussion}

The present study evaluated the effect of a combination of ZOL implant-coating with systemic bone anabolic sclerostin antibody treatment versus uncoated reference implants with sclerostin antibody

a

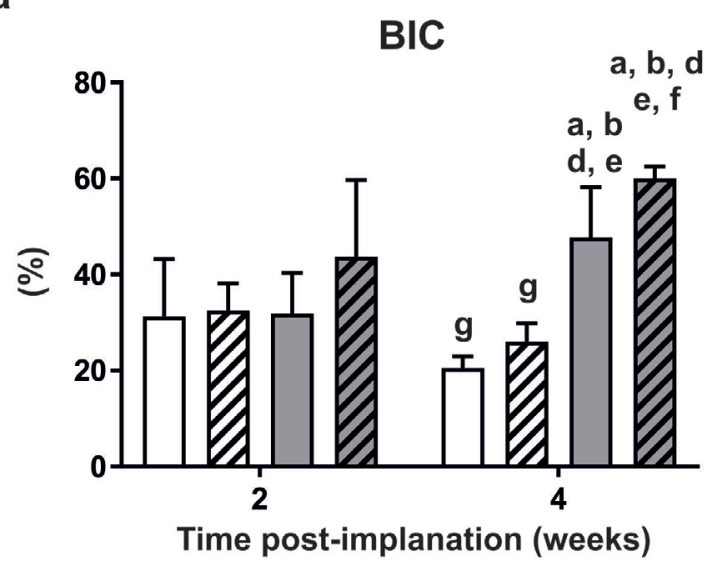

C

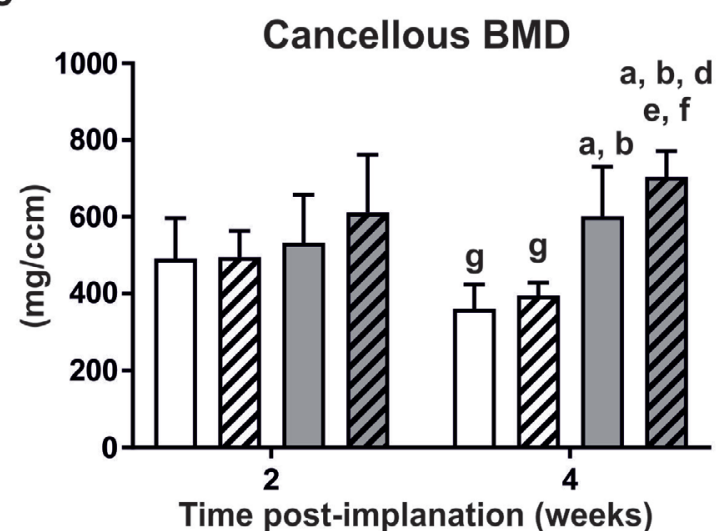

Reference coating without sclerostin antibody

Reference coating with sclerostin antibody treatment or ZOL implant-coating only in a rat osteoporosis model. The study hypothesis was that the combination of sclerostin antibody application and ZOL coating promoted more osseointegration than either agent alone. This could be verified by histomorphometrical and mechanical testing.

After 4 weeks of healing, sclerostin antibody therapy combined with ZOL implant-coating led to a significant increase in BIC, BA/TA adjacent to the implant, cancellous $\mathrm{BV} / \mathrm{TV}$ and $\mathrm{BMD}$ as well as removal torque as compared to animals receiving only sclerostin antibody treatment or only ZOL implant-coating. Likewise, the dynamics of peri-implant bone deposition differed. Only the combination treatment induced sustained periimplant bone deposition displayed by rising BIC and BA/TA values over time between 2 and 4 weeks post-implantation. This significant increase could be

b

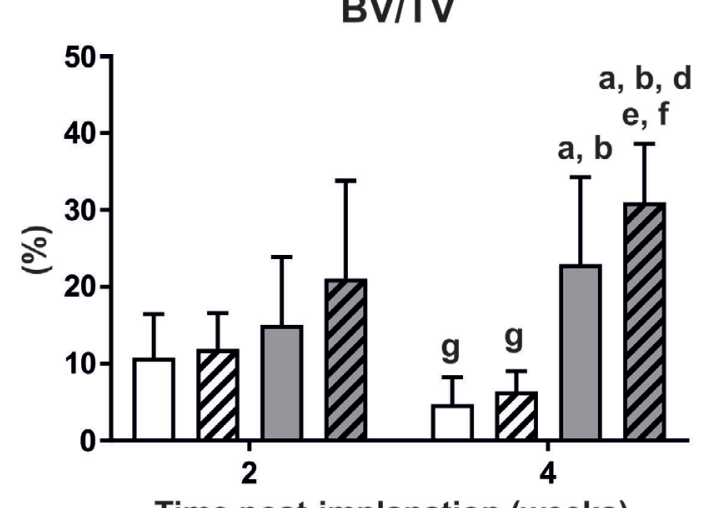

Time post-implanation (weeks)

d

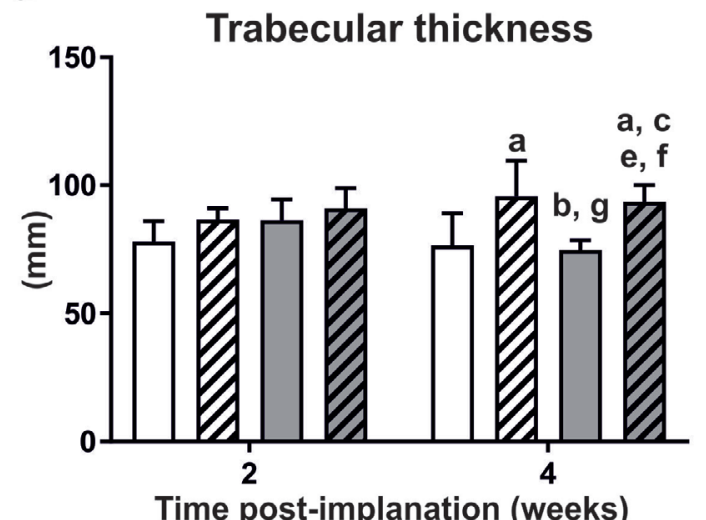

ZOL coating without sclerostin antibody

ZOL coating with sclerostin antibody

Fig. 5. $\mu \mathrm{CT}$ parameters 2 and 4 weeks post-implantation. (a) BIC, (b) BV/TV, (c) cancellous BMD and (d) trabecular thickness were measured in an area of $200 \mu \mathrm{m}$ surrounding the implant. Statistical significance is designated as follows: ${ }^{a} p<0.05$ versus reference coating without sclerostin antibody at same time point; ${ }^{\mathrm{b}} p<0.01$ versus reference coating with sclerostin antibody at same time point; ${ }^{c} p<0.01$ versus ZOL coating without sclerostin antibody at same time point; ${ }^{\mathrm{d}} p<0.05$ versus reference coating without sclerostin antibody at 2 weeks post-implantation; ${ }^{\mathrm{e}} p<0.05$ versus reference coating with sclerostin antibody at 2 weeks postimplantation; ${ }^{\mathrm{f}} p<0.001$ versus ZOL coating without sclerostin antibody at 2 weeks post-implantation; g $p<0.05$ versus ZOL coating with sclerostin antibody at 2 weeks post-implantation. 
explained by a complementary mechanism of action of both pharmacologic agents. ZOL primarily has an anti-resorptive function due to osteoclast inhibition (Im et al., 2004), thus maintaining the peri-implant bone. Secondarily, bisphosphonates can also elicit an osteoanabolic effect (Im et al., 2004). Conversely, sclerostin antibody acts mainly as osteoanabolic, by increasing osteoblastic lamellar bone formation on existing bone surfaces, but can also exert antiresorptive effects (McClung, 2017). Therefore, the combination of both agents is expected to result in a strong anti-resorptive effect in the peri-implant bone area and an osteoanabolic effect in the surrounding bone tissue. This can be clinically associated with an improved osseointegration of orthopaedic or dental implants (Virdi et al., 2012; Virdi et al., 2015).

The effect of sclerostin antibody application on implant osseointegration and bone healing has been previously assessed in small animal models. Agholme et al. (2010) analysed the pull-out strength of metaphyseal inserted steel screws in a rat model after 2 and 4 weeks. A similar study was performed by Virdi et al. (2012). The insertion of acid-etched implants into the medullar canal of murine femora combined with sclerostin antibody injection twice a week led to an increased mechanical fixation of the implants in accordance with Agholme et al. (2010). Based on these promising results in noncompromised bone tissue, subsequent studies used animal models simulating systemic bone diseases, such as osteoporosis. OVX rats are a standard model of postmenopausal osteoporosis (Thompson et al.,
1995). Similarly to the present study, Virdi et al. (2015) used this model to quantify the improvement of implant fixation caused by sclerostin antibody treatment. Titanium implants were inserted into the femoral medullar cavity of OVX rats. A sclerostin antibody was administered twice per week (25 mg/ $\mathrm{kg}$ ) for 4,8 or 12 weeks. When compared to an OVX control group, antibody treatment led to enhanced BIC, peri-implant trabecular thickness and volume, as well as increased cortical thickness. The stimulation of bone formation was also detectable in the present study, but to a lesser extent. In comparison with implants placed into oestrogen-competent rat, the antibody could not fully compensate for the effect of ovariectomy on bone architecture (Stadlinger et al., 2013). One possible explanation could be the promotion of lamellar bone formation by antibody treatment solely on existing trabeculae (Virdi et al., 2015). Furthermore, the impact of the healing phase has to be considered. The most pronounced effect of the sclerostin antibody can be expected during the bone remodelling phase following implantation (Virdi et al., 2012). In case of OVX rats, a remodelling time between 4 and 8 weeks post-implantation is reported (Virdi et al., 2012). Therefore, a 2 weeks observation period following implantation, as in the present study, might be too short to display the whole therapeutic potential of the sclerostin antibody.

Bisphosphonates can be administered in various ways. In animal studies analysing implant osseointegration, systemic administration by subcutaneous (Viera-Negron et al., 2008) or a

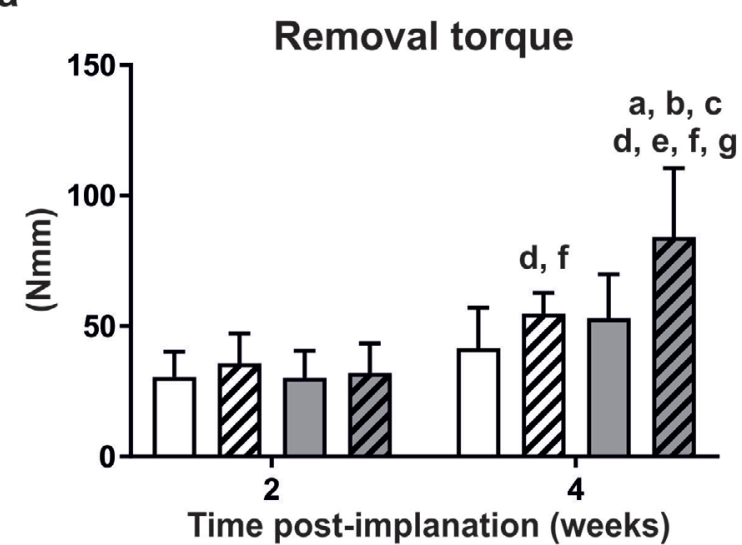

Reference coating without sclerostin antibody Reference coating with sclerostin antibody b

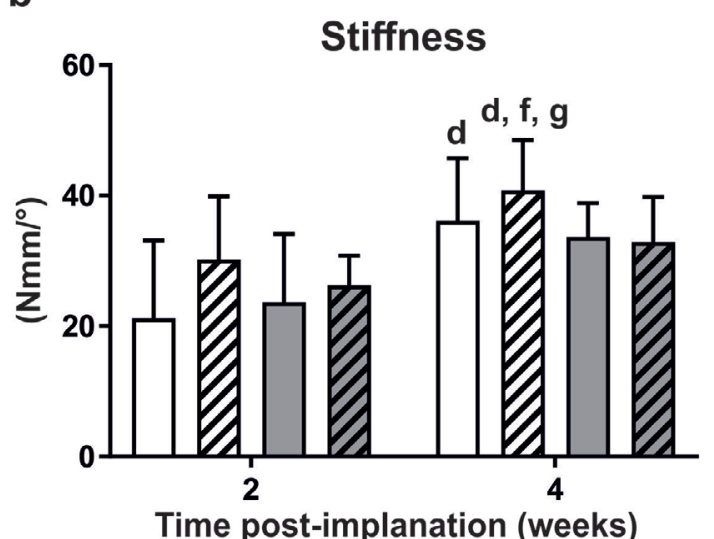

ZOL coating without sclerostin antibody

ZOL coating with sclerostin antibody

Fig. 6. Mechanical testing of implant osseointegration 2 and 4 weeks post-implantation. (a) Removal torque and (b) stiffness were quantified. Statistical significance is designated as follows: ${ }^{a} p<0.0001$ versus reference coating without sclerostin antibody at same time point; ${ }^{\mathrm{b}} p<0.01$ versus reference coating with sclerostin antibody at same time point; ${ }^{c} p<0.001$ versus ZOL coating without sclerostin antibody at same time point; ${ }^{\mathrm{d}} p<0.05$ versus reference coating without sclerostin antibody at 2 weeks post-implantation; ${ }^{\mathrm{e}} p<0.0001$ versus reference coating with sclerostin antibody at 2 weeks post-implantation; ${ }^{\mathrm{f}} p<0.05$ versus ZOL coating without sclerostin antibody at 2 weeks post-implantation; ${ }^{\mathrm{g}} p<0.05$ versus ZOL coating with sclerostin antibody at 2 weeks post-implantation. 
intravenous (Yildiz et al., 2010) administration routes as well as the application as an implant coating component (Gao et al., 2009; Langhoff et al., 2008; Wermelin and Suska et al., 2008) have been previously reported. Studies analysing bisphosphonate containing implant coatings have shown a local effect of the agent in an area of about $1 \mathrm{~mm}$ within the periimplant tissue region (Abtahi et al., 2010; Wermelin and Suska et al., 2008). Thus, the therapeutic effect appears to take place only within the clinically relevant peri-implant area and, therefore, may reduce or avoid possible systemic side effects. Up to now, osteonecrosis of the jaw has not been described to occur surrounding bisphosphonate-coated implants (Abtahi et al., 2010). Nevertheless, safety aspects have to be considered, especially prior to clinical studies using bisphosphonate-coated implants. Different bisphosphonates such as alendronic acid or ZOL show differences in therapeutic potential and pharmacologic profile due to their specific chemical structure. ZOL probably has the highest potential to promote osseointegration in OVX-rats (Andersson et al., 2010; Gao et al., 2009). ZOL-coated implant surfaces have been previously used in preclinical studies in OVX rats (Gao et al., 2009; Stadlinger et al., 2013). Gao et al. (2009) measured significantly increased BIC and BV/TV values after 12 weeks of healing with the bisphosphonate being detectable on the implant surface up to $21 \mathrm{~d}$ following surgery. The same effect on bone formation could be observed using a lower ZOL concentration. Interestingly, a significant effect on peri-implant bone healing is not detectable until the fourth week of healing, although surface release kinetics in vitro show a release of ZOL within less than $21 \mathrm{~d}$ (Stadlinger et al., 2013). Therefore, the sustained therapeutic effect of bisphosphonate coatings may be explained by the local recycling of the pharmaceutical agent. After 4 weeks of healing, a BIC of $43.2 \pm 17.8 \%$ in OVX rats receiving $\mathrm{ZOL}$-coated implants was measured by histomorphometry. In comparison, the additional application of sclerostin antibody in the present study lead to a BIC of $57.4 \pm 15.0 \%$, demonstrating an increased therapeutic benefit. Moreover, this further increase almost reached the level of the nonOVX reference group as reported by Stadlinger et al. (2013). Cancellous BV/TV adjacent and distant to the implant were similarly increased, emphasising the pharmacological potential of a combined application of sclerostin antibody and ZOL coating on periimplant bone formation. The concept of an add-on therapy to increase bone mass in osteoporotic bone and, thus, to improve implant osseointegration has already been described by Li et al. (2011). In OVX rats with established osteopaenia, the bisphosphonate alendronic acid and/or sclerostin antibody were systemically applied. After 12 weeks, increased trabecular bone volume and bone strength were measured in all antibody-treated groups irrespective of the systemic alendronic acid application ( $\mathrm{Li}$ et al., 2011). Halleux et al. (2009) analysed the effect of
ZOL and sclerostin antibody combination therapy on bone mass in OVX mice treated immediately after ovariectomy. In this setting of active bone loss, loss was prevented and bone mass was increased to a greater extent when using the combination therapy as compared to either agent alone.

\section{Limitations of the study}

In the field of dental implant research, small and large animal models are well-established (Berglundh et al., 2012). Histological analyses, which are necessary to evaluate new implant modifications, are easier to perform in preclinical models than in clinical settings. Stadlinger et al. (2012b) reviewed the animal models applied in 271 preclinical dental implant studies. Almost half of the studies were performed in small animal models (123 studies; $45.4 \%$ ). Especially, for the first reported in vivo study of a new dental implant or implant modification, small animals are the model of choice. Rats and rabbits are the most commonly used small animals in dental implantology (Dereka et al., 2018; Stadlinger et al., 2012b). In rats, symptoms of postmenopausal osteoporosis can be induced by ovariectomy with well-known phenotypic consequences of strongly enhanced osteoclastic bone resorption (Kimmel, 2002; Komori 2015).

The distribution of implant sites was also analysed by Stadlinger et al. (2012b). Irrespective of the selected animal model, mainly extraoral implant sites are used $(61 \%)$ for which the tibia is the most common location. In small animals, such as rats, the application of implants with an adequate size is much easier using extraoral sites, because no tooth extraction is required before implant insertion and often there is more space for surgical intervention. In addition, small animals cannot be trained for oral hygiene and intraoral wound protection after surgery, which might influence implant healing. The disadvantage of extraoral versus intraoral implant insertion is the missing interaction of the oral microbiome as well as the mechanical strain from the chewing forces on the osseointegration process. Nevertheless, the tibia is also mechanically loaded by the natural movement of the rat and is amenable to controlled loading, allowing the study of the effects of mechanical load on implant healing (Duyck and Vandamme, 2014).

The different embryological origin of craniofacial and long bones, such as the tibia, need to be addressed. Neural crest cells represent a multipotent cell population which undergo an epithelialmesenchymal transition during migration from the neural tube, building the jaw bones through intramembranous ossification (Noce et al., 2014). In contrast, long bones are of paraxial mesodermal origin and develop through endochondral ossification. The scientific community is still debating if the source of stem cells for mineralised tissue regeneration in the craniomaxillofacial region might influence the repairing result (d'Aquino et al., 2011). Alge et al. (2010) concluded that dental pulp stem cells are more suitable for craniomaxillofacial repair than 
bone marrow stem cells because of the differing embryological origin. Therefore, it is possible that the chosen implant site also has an impact, in terms of differing embryological origin, on the osseous healing after implant insertion. Nevertheless, when comparing the overall structure of jaw and tibial bone, dense cortical bone surrounds a cancellous bone compartment, suggesting overall comparable implant sites. The focus of the present study was the evaluation of implant healing and osseointegration within the first 4 weeks of bone healing. Future studies will need to evaluate the long-term effects of bisphosphonate coating and sclerostin antibody administration.

Yu et al. (2018) used a non-osteoporotic experimental alveolar ridge tooth extraction rat model and administered the antibody starting on the day of surgery. Sclerostin-antibody-treated and control groups were sacrificed after 10, 14 and $28 \mathrm{~d}$ and their maxillae were evaluated by $\mu \mathrm{CT}$, histology and histomorphometry. Results indicated that systemic sclerostin antibody administration enhanced osseointegration and bone regeneration around dental implants also at compromised alveolar bone osteotomy sites. It was concluded that the approach offers potential as a treatment modality for patients with low bone mass or bone defects to achieve a more predictable bone regeneration at alveolar bone defects and to enhance dental implant osseointegration. This is in line with the present study results. In addition, findings demonstrated that, in an osteoporosis model, the beneficial effect of osteoanabolic sclerostin antibody therapy can be further increased by using a ZOL implant-coating.

The present preclinical study showed promising results in a rat model of postmenopausal osteoporosis regarding the stimulation of implant osseointegration in a compromised bone setting. While promising, these results should still be validated in a larger animal model, e.g. the osteoporotic minipig, in which dental implants can be inserted intraorally (Stadlinger et al., 2012b). Currently, ZOL, like all other bisphosphonates, is clinically approved but not licensed as a dental implant coating. Recently, the anti-sclerostin antibody romosozumab has been approved by the Japanese Pharmaceuticals and Medical Devices Agency and is under review with the European Medicines Agency and the US Food and Drug Administration for the treatment of osteoporotic patients at high fracture risk. The question whether systemic sclerostin antibody therapy is also justifiable merely to increase implant osseointegration needs to be carefully addressed. Consensus is that the oral reconstruction after tooth loss is necessary to restore masticatory, speech and aesthetic functions of the patient. The dental reconstruction can be performed by removable and non-removable dental prostheses. Especially in cases of complete tooth loss, the stable fixation of the prosthesis is limited due to the anatomy of the edentulous jaw. The patients benefit greatly from fixation of the prosthesis by dental implants, because the ability to eat normally is not just important from the medical point of view, but it also increases the patients' overall well-being and satisfaction. Because of the demographic development in the industrial nations, the number of aging and diseased patients with compromised osteoporotic bone requiring treatment is expected to increase rapidly in the future. For these patients with compromised bone, systemic osteoanabolic principles, such as sclerostin antibody therapy in combination with implant coatings, might be one way to benefit from the advantages of a fixed denture. Nevertheless, careful consideration of the risks and possible benefits for the patient remains to be always undertaken. It is also conceivable that a sustained-release formulation of sclerostin antibody can be developed for local use in patients with compromised bone in order to enhance dental implant osseointegration. To ensure the long-term success of such a local application strategy, more preclinical dose-regimen studies are needed to better understand during which phase of implant wound healing and osseointegration the osteoanabolic properties of the anti-sclerostin therapy are contributing most. Likely, local application of sclerostin antibody would require sustained drug release over time, which might be achieved by sclerostin antibody implant coating. From a technical point of view, this may be challenging, especially if two different components such as a sclerostin antibody and ZOL shall be combined.

Dental implants enable functional and aesthetic rehabilitation after tooth loss. However, compromised host bone can be a limitation for implant placement and the clinical long-term success may be reduced. Therefore, it is of interest to promote peri-implant bone formation using implant coatings and to restore adjacent cancellous bone mass and structure with a systemic bone anabolic agent in patients at risk. In the current study, the combination of anti-resorptive ZOL implant coating and systemically applied sclerostin antibody led to significantly increased BIC, BV/TV, $\mathrm{BA} / \mathrm{TA}, \mathrm{BMD}$ and removal torque in an osteoporotic rat model after 4 weeks of healing as compared to rats only receiving reference coated implants and sclerostin antibody treatment. Therefore, the combination of ZOL and osteoanabolic sclerostin antibody has the potential to be more effective than either agent alone.

\section{Acknowledgement}

This study was co-founded by Novartis Pharma AG, Thommen Medical AG and the German Research Foundation "SFB Transregio 67" project. The authors would like to thank Peter Ingold, Marcel Merdes, Marco Pegurri, Renzo Schumpf, Andrea Venturiere and Anne Studer for their excellent technical support.

Ina Kramer and Micheala Kneissel are employees of Novartis Pharma AG. Falko Schlottig was 
employed by Thommen Medical at the time of the study. All other authors have no conflict of interest to declare.

\section{References}

Abtahi J, Tengvall P, Aspenberg P (2010) Bisphosphonate coating might improve fixation of dental implants in the maxilla: a pilot study. Int J Oral Maxillofac Surg 39: 673-677.

Agholme F, Li X, Isaksson H, Ke HZ, Aspenberg P (2010) Sclerostin antibody treatment enhances metaphyseal bone healing in rats. J Bone Miner Res 25: 2412-2418.

Alge DL, Zhou D, Adams LL, Wyss BK, Shadday MD, Woods EJ, Gabriel Chu TM, Goebel WS (2010). Donor-matched comparison of dental pulp stem cells and bone marrow-derived mesenchymal stem cells in a rat model. J Tissue Eng Regen Med 4: 73-81.

Andersson T, Agholme F, Aspenberg P, Tengvall P (2010) Surface immobilized zoledronate improves screw fixation in rat bone: a new method for the coating of metal implants. J Mater Sci Mater Med 21: 3029-3037.

Baron R, Kneissel M (2013) WNT signaling in bone homeostasis and disease: from human mutations to treatments. Nat Med 19: 179-192.

Berglundh T, Stavropoulos A (2012) Preclinical in vivo research in implant dentistry. Consensus of the eighth European workshop on periodontology. J Clin Periodontol 39 Suppl 12: 1-5.

Branemark PI, Hansson BO, Adell R, Breine U, Lindstrom J, Hallen O, Ohman A (1977) Osseointegrated implants in the treatment of the edentulous jaw. Experience from a 10-year period. Scand J Plast Reconstr Surg 16: 1-132.

Crandall CJ, Newberry SJ, Diamant A, Lim Y-W, Gellad WF, Booth MJ, Motala A, Shekelle PG (2014) Comparative effectiveness of pharmacologic treatments to prevent fractures: an updated systematic review. Ann Intern Med 161: 711-723.

d'Aquino R, Tirino V, Desiderio V, Studer M, De Angelis GC, Laino L, De Rosa A, Di Nucci D, Martino S, Paino F, Sampaolesi M, Papaccio G (2011). Human neural crest-derived postnatal cells exhibit remarkable embryonic attributes either in vitro or in vivo. Eur Cell Mater 21: 304-316.

Delgado-Calle J, Sato AY, Bellido T (2017) Role and mechanism of action of sclerostin in bone. Bone 96: 29-37.

Donath K, Breuner G (1982) A method for the study of undecalcified bones and teeth with attached soft tissues. The Sage-Schliff (sawing and grinding) technique. J Oral Pathol 11: 318-326.

Duarte PM, Vasconcelos Gurgel BC de, Sallum AW, Filho GRN, Sallum EA, Nociti FH, JR (2005) Alendronate therapy may be effective in the prevention of bone loss around titanium implants inserted in estrogen-deficient rats. J Periodontol 76: 107-114.
Duyck J and Vandamme K (2014) The effect of loading on peri-implant bone: a critical review of the literature. J Oral Rehab 41: 783-794.

Gao Y, Luo E, Hu J, Xue J, Zhu S, Li J (2009) Effect of combined local treatment with zoledronic acid and basic fibroblast growth factor on implant fixation in ovariectomized rats. Bone 44: 225-232.

Halleux C, Hu S, Diefenbach B, Prassler J, Merdes M, Studer A, Heine H, Hartmann S, Mackenzie A, Gamse R, Junker U, Kneissel M (2009) Infrequent cotreatment abd sequential treatment of anti-sclerostin antibody with zoledronic acid restores and maintains bone mass in murine osteoporosis models. J Bone Miner Res 24 Suppl 1. Abstract 1063. DOI: 10.1002/ jbmr.5650241301.

Haussler B, Gothe H, Gol D, Glaeske G, Pientka L, Felsenberg D (2007) Epidemiology, treatment and costs of osteoporosis in Germany-the BoneEVA Study. Osteoporos Int 18: 77-84.

Holahan CM, Koka S, Kennel KA, Weaver AL, Assad DA, Regennitter FJ, Kademani D (2008) Effect of osteoporotic status on the survival of titanium dental implants. Int J Oral Maxillofac Implants 23: 905-910.

Ihde S, Kopp S, Maier T (2009) Comparison of implant survival with implants placed in acceptable and compromised bone: a literature review. J Maxillofac Oral Surg 8: 1-7.

Im G-I, Qureshi SA, Kenney J, Rubash HE, Shanbhag AS (2004) Osteoblast proliferation and maturation by bisphosphonates. Biomaterials 25: 4105-4115.

Jeffcoat MK (2006) Safety of oral bisphosphonates: controlled studies on alveolar bone. Int J Oral Maxillofac Implants 21: 349-353.

Kimmel DB (2002) Principles of Bone Biology: Animal Models in Osteoporosis Research, Academic Press, London, pp 1635-1655.

Komori T (2015) Animal models for osteoporosis. Eur J Pharmacol 759: 287-294.

Langhoff JD, Voelter K, Scharnweber D, Schnabelrauch M, Schlottig F, Hefti T, Kalchofner K, Nuss K, Rechenberg B von (2008) Comparison of chemically and pharmaceutically modified titanium and zirconia implant surfaces in dentistry: a study in sheep. Int J Oral Maxillofac Surg 37: 1125-1132.

La Noce M, Mele L, Tirino V, Paino F, De Rosa A, Naddeo P, Papagerakis P, Papaccio G, Desiderio V (2014) Neural crest stem cell population in craniomaxillofacial development and tissue repair. Eur Cell Mater 28: 348-357.

Leupin O, Piters E, Halleux C, Hu S, Kramer I, Morvan F, Bouwmeester T, Schirle M, Bueno-Lozano M, Fuentes FJR, Itin PH, Boudin E, Freitas F de, Jennes K, Brannetti B, Charara N, Ebersbach H, Geisse S, Lu CX, Bauer A, van Hul W, Kneissel M (2011) Bone overgrowth-associated mutations in the LRP4 gene impair sclerostin facilitator function. J Biol Chem 286: 19489-19500.

Li X, Ominsky MS, Warmington KS, Niu Q-T, Asuncion FJ, Barrero M, Dwyer D, Grisanti M, Stolina 
M, Kostenuik PJ, Simonet WS, Paszty C, Ke HZ (2011) Increased bone formation and bone mass induced by sclerostin antibody is not affected by pretreatment or cotreatment with alendronate in osteopenic, ovariectomized rats. Endocrinology 152: 3312-3322.

Lin JH (1996) Bisphosphonates: a review of their pharmacokinetic properties. Bone 18: 75-85.

Looker AC (1995) Prevalence of low femoral bone density in older U.S. women from NHANES III. J Bone Miner Res 10: 796-802.

Maimoun L, Brennan TC, Badoud I, DuboisFerriere V, Rizzoli R, Ammann P (2010) Strontium ranelate improves implant osseointegration. Bone 46: 1436-1441.

Marco F, Milena F, Gianluca G, Vittoria O (2005) Peri-implant osteogenesis in health and osteoporosis. Micron 36: 630-644.

McClung MR (2017) Sclerostin antibodies in osteoporosis: latest evidence and therapeutic potential. Ther Adv Musculoskelet Dis 9: 263-270.

Ominsky MS, Boyce RW, Li X, Ke HZ (2017) Effects of sclerostin antibodies in animal models of osteoporosis. Bone 96: 63-75.

Poole KES, van Bezooijen RL, Loveridge N, Hamersma H, Papapoulos SE, Lowik CW, Reeve J (2005) Sclerostin is a delayed secreted product of osteocytes that inhibits bone formation. FASEB J 19: 1842-1844.

Qi MC, Zhou XQ, Hu J, Du ZJ, Yang JH, Liu M, Li XM (2004) Oestrogen replacement therapy promotes bone healing around dental implants in osteoporotic rats. Int J Oral Maxillofac Surg 33: 279-285.

Rebaudi A, Koller B, Laib A, Trisi P (2004) Microcomputed tomographic analysis of the periimplant bone. Int J Periodontics Restorative Dent 24: 316-325.

Scully C, Hobkirk J, Dios PD (2007) Dental endosseous implants in the medically compromised patient. J Oral Rehabil 34: 590-599.

Shirota T, Tashiro M, Ohno K, Yamaguchi A (2003) Effect of intermittent parathyroid hormone (1-34) treatment on the bone response after placement of titanium implants into the tibia of ovariectomized rats. J Oral Maxillofac Surg 61: 471-480.

Stadlinger B, Bierbaum S, Grimmer S, Schulz MC, Kuhlisch E, Scharnweber D, Eckelt U, Mai R (2009) Increased bone formation around coated implants. J Clin Periodontol 36: 698-704.

Stadlinger B, Ferguson SJ, Eckelt U, Mai R, Lode AT, Loukota R, Schlottig F (2012a) Biomechanical evaluation of a titanium implant surface conditioned by a hydroxide ion solution. Br J Oral Maxillofac Surg 50: 74-79.

Stadlinger B, Pourmand P, Locher MC, Schulz MC (2012b) Systematic review of animal models for the study of implant integration, assessing the influence of material, surface and design. J Clin Periodontol 39 Suppl 12: 28-36.

Stadlinger B, Korn P, Todtmann N, Eckelt U, Range U, Burki A, Ferguson SJ, Kramer I, Kautz A, Schnabelrauch M, Kneissel M, Schlottig F (2013)
Osseointegration of biochemically modified implants in an osteoporosis rodent model. Eur Cell Mater 25: 326-340.

Stadlinger B, Pilling E, Mai R, Bierbaum S, Berhardt R, Scharnweber D, Eckelt U (2008) Effect of biological implant surface coatings on bone formation, applying collagen, proteoglycans, glycosaminoglycans and growth factors. J Mater Sci Mater Med 19: 1043-1049.

Thompson DD, Simmons HA, Pirie CM, Ke HZ (1995) FDA guidelines and animal models for osteoporosis. Bone 17: 125S-133S.

Viera-Negron YE, Ruan W-h, Winger JN, Hou X, Sharawy MM, Borke JL (2008) Effect of ovariectomy and alendronate on implant osseointegration in rat maxillary bone. J Oral Implantol 34: 76-82.

Virdi AS, Irish J, Sena K, Liu M, Ke HZ, McNulty MA, Sumner DR (2015) Sclerostin antibody treatment improves implant fixation in a model of severe osteoporosis. J Bone Joint Surg Am 97: 133-140.

Virdi AS, Liu M, Sena K, Maletich J, McNulty M, Ke HZ, Sumner DR (2012) Sclerostin antibody increases bone volume and enhances implant fixation in a rat model. J Bone Joint Surg Am 94: 1670-1680.

Wermelin K, Aspenberg P, Linderback P, Tengvall P (2008a) Bisphosphonate coating on titanium screws increases mechanical fixation in rat tibia after two weeks. J Biomed Mater Res A 86: 220-227.

Wermelin K, Suska F, Tengvall P, Thomsen P, Aspenberg P (2008b) Stainless steel screws coated with bisphosphonates gave stronger fixation and more surrounding bone. Histomorphometry in rats. Bone 42: 365-371.

Wermelin K, Tengvall P, Aspenberg P (2007) Surface-bound bisphosphonates enhance screw fixation in rats--increasing effect up to 8 weeks after insertion. Acta Orthop 78: 385-392.

Yildiz A, Esen E, Kurkcu M, Damlar I, Daglioglu K, Akova T (2010) Effect of zoledronic acid on osseointegration of titanium implants: an experimental study in an ovariectomized rabbit model. J Oral Maxillofac Surg 68: 515-523.

Yu SH, Hao J, Fretwurst T, Liu M, Kostenuik P, Giannobile WV, Jin Q (2018) Sclerostin-neutralizing antibody enhances bone regeneration around oral implants. Tissue Eng Part A 24: 1672-1679.

\section{Discussion with Reviewer}

Babak Chehroudi: Implant coatings are often unstable in post-surgical environments where abundance of proteolytic enzyme and inflammatory cells can destabilise them rapidly. Did some of the long-term results of this experiment suggest that the implant coating was removed? How long materials such as ZOL remain on the implant surface in the harsh post-trauma environment?

Authors: Stadlinger et al. (2013) showed an in vitro ZOL-coating release of $71.1 \pm 4.7 \%$ after $14 \mathrm{~d}$. This is in accordance with the findings of Gao et al. (2009), who showed a release of bisphosphonates 
from the implant surface over a period of $21 \mathrm{~d}$. Clinically, bisphosphonates such as ZOL are taken up by osteoclasts and, after apoptosis of the cell, the drug is released again into the extracellular matrix. Subsequently, the bisphosphonate can again be absorbed by osteoclasts (Li, 1996). The local recycling of ZOL leads to a very long half-life of this class of drugs, differently from protein implant coatings with e.g. extracellular matrix components. Therefore, the long-term effectiveness of bisphosphonates as implant coatings might not only be determined by the initial drug release kinetics.

Editor's note: The Scientific Editor responsible for this paper was Thimios Mitsiadis. 